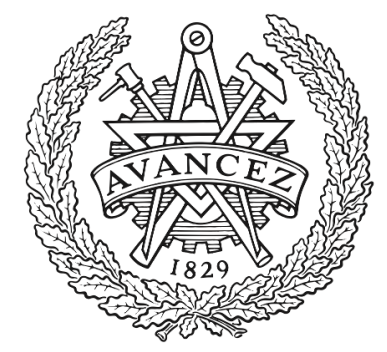

\title{
CHALMERS
}

UNIVERSITY OF TECHNOLOGY

\section{A Nonlinear Transmission Line Model for Simulating Distributed SIS Frequency Multipliers}

Downloaded from: https://research.chalmers.se, 2023-04-26 12:02 UTC

Citation for the original published paper (version of record):

Garrett, J., Marouf Rashid, H., Yassin, G. et al (2020). A Nonlinear Transmission Line Model for Simulating Distributed SIS Frequency Multipliers. IEEE Transactions on Terahertz Science and Technology, 10(3): 246-255. http://dx.doi.org/10.1109/TTHZ.2020.2979125

N.B. When citing this work, cite the original published paper.

(O2020 IEEE. Personal use of this material is permitted.

However, permission to reprint/republish this material for advertising or promotional purposes 


\title{
A Nonlinear Transmission Line Model for Simulating Distributed SIS Frequency Multipliers
}

\author{
John D. Garrett, Hawal Rashid, Ghassan Yassin, Vincent Desmaris, Member, IEEE, Alexey Pavolotsky, and \\ Victor Belitsky, Senior Member, IEEE
}

\begin{abstract}
Superconductor / Insulator / Superconductor (SIS) junctions have extremely nonlinear electrical properties, which makes them ideal for a variety of applications, including heterodyne mixing and frequency multiplication. With SIS mixers, the SIS junctions normally have circular cross-sections, but they can also be fabricated in the form of microstrip transmission lines, known as distributed SIS junctions (DSJs). By using a DSJ as an open-circuit stub, it is possible to create a large SIS junction with a low effective input reactance. This is beneficial for SIS frequency multipliers because their output power is proportional to the area of the junction. It is challenging, however, to simulate the behavior of DSJs because (a) they have to be modeled as transmission lines and (b) the model has to take into account the quasiparticle tunneling current, which is a nonlinear function of the $\mathrm{AC}$ voltage. In this paper, we present a new nonlinear transmission line model to accurately describe the behavior of DSJs and to simulate the performance of distributed SIS frequency multipliers (DSMs). This model is compared to experimental data from a recent DSM device and good agreement is found between the DC tunneling currents and the output powers at the second harmonic. Based on this success, an improved DSM design is proposed that has a higher output power and a higher conversion efficiency than previous designs.
\end{abstract}

Index Terms-Superconductor/Insulator/Superconductor (SIS) junctions, distributed SIS junctions, frequency multiplication, nonlinear transmission line models, terahertz receivers, superconducting detectors

\section{INTRODUCTION}

At millimeter and submillimeter wavelengths, radio telescopes use Superconductor / Insulator / Superconductor (SIS) junctions to build extremely sensitive heterodyne receivers. These receivers are now in widespread use and modern SIS mixers have noise properties that are only 2 to 3 times the quantum limit [1]-[3]. Current research is now seeking to develop SIS receivers that operate above $1 \mathrm{THz}$ and focal plane arrays that allow multiple SIS receivers to observe simultaneously. Both of these applications require powerful local-oscillator (LO) signals in order to drive the SIS junctions and efficiently down-convert the astronomical signals.

Manuscript received July 5, 2019; revised December 21, 2019 and February 10, 2020; accepted February 19, 2020.

J. Garrett was with the Department of Physics (Astrophysics), University of Oxford, Oxford, U.K. He is now with the Center for Astrophysics | Harvard \& Smithsonian, Cambridge 02140, MA (e-mail: john.garrett@cfa.harvard.edu).

H. Rashid is with the Chalmers University of Technology, Gothenburg, Sweden, and also with ANSYS Sweden AB, Gothenburg, Sweden.

G. Yassin is with the Department of Physics (Astrophysics), University of Oxford, Oxford, U.K.

V. Desmaris, A. Pavolotsky and V. Belitsky are with the Chalmers University of Technology, Gothenburg, Sweden.
In most LO sources, the signal is initially generated by a synthetic signal generator, a Gunn diode or an yttriumiron-garnet (YIG) oscillator. The frequency of the signal is then multiplied to the desired value, often in several stages, using nonlinear components. For example, the Atacama Large Millimeter Array (ALMA) Band-9 LO uses a $\times 3 \times 3 \times 3$ multiplier chain to produce a $600-720 \mathrm{GHz}$ signal [2], [4] and the ALMA Band- 10 LO uses a $\times 2 \times 3 \times 3 \times 3$ multiplier chain to produce a 792-945 GHz signal [4], [5]. (Both of these LO sources use YIG oscillators to generate the initial input signals.) With several cascaded frequency multipliers, it is then very important that each multiplier within the $\mathrm{LO}$ chain has a high conversion efficiency in order to produce an adequate output power for the SIS receiver. This is especially true for receivers that operate above $1 \mathrm{THz}$, which require additional frequency multiplication stages, and for focal plane arrays, which split the LO signal between multiple SIS devices.

Currently, most frequency multipliers use Schottky diodes to generate higher-order harmonics from sinusoidal input signals; however, recent research has proposed using SIS junctions instead [6]-[9]. Since SIS junctions have extremely nonlinear properties, these multipliers may be able to provide higher conversion efficiencies and operate at higher frequencies than Schottky diodes. SIS frequency multipliers also have the added advantage that they can be easily integrated with the SIS mixers inside the cryostat, which would reduce the complexity of the receiver and the loss in the LO signal path. For instance, in the current ALMA Band-5 receiver, the final frequency doubler is placed inside the cryostat on the $110 \mathrm{~K}$ stage [1], [4]. By using an SIS frequency multiplier, this component could be integrated with the SIS mixer on the $4 \mathrm{~K}$ stage, potentially even on a single chip.

The challenge with SIS frequency multipliers is that their output power is proportional to the area of the junction (assuming a constant tunneling current density). Unfortunately, the intrinsic capacitance of the junction is also proportional to the area, which could severely limit the bandwidth of the multiplier if it is not tuned sufficiently. To overcome this obstacle, SIS frequency multipliers were developed using arrays of smaller SIS junctions connected in series [6], but these multipliers suffered from local heating effects and inter-mixing between the harmonics. Rashid et al. [7]-[9] then reported a new frequency multiplier using distributed SIS junctions (DSJs), which are SIS junctions fabricated in the form of microstrip transmission lines. By using DSJs as open-circuit stubs, it is possible to reduce the effective input reactance, allowing DSJs to be much larger than circular junctions, while 
maintaining a favorable input impedance. Unlike series arrays, DSJs also do not encounter the same issues with heating and inter-mixing [6]-[9].

To simulate the performance of distributed SIS frequency multipliers (DSMs), Rashid et al. [7]-[9] presented a quasinonlinear transmission line model and compared it to their experimental DSM device. They found reasonable agreement, but the model did not take into account that the tunneling current and the propagation constant should change with position along the DSJ, depending on the magnitude of the AC voltage. To address this limitation, we now present in this paper a new fully nonlinear transmission line model, which should improve the accuracy of DSJ simulations. This model divides the DSJ into a series of segments that are small enough that the propagation constant can be assumed to be fixed over each unit length. It then uses spectral-domain simulation software to calculate the nonlinear response of the SIS junction and estimate the power generated at the second harmonic. In the following, the quasi-nonlinear model is reviewed in Sec. II, the new nonlinear model is presented in Sec. III, and then it is compared to experimental data in Sec. IV. Lastly, using the new nonlinear model, an improved DSM device is proposed in Sec. V, which has both higher output power and higher efficiency than current designs. In future work, we hope to fabricate this new DSM device and further validate the model by comparing it to additional experimental data. This initial paper will focus on the limitations of the quasinonlinear model and how these limitations are addressed by the new nonlinear DSJ model.

\section{QUASI-NONLINEAR DSJ TRANSMISSION LINE MODEL}

A diagram of a DSJ is shown in Fig. 1a. It is characterized by its width $W$ and length $L$, as well as the thickness of the wiring, insulation and ground layers, represented by $d_{\mathrm{w}}$, $d_{\mathrm{i}}$ and $d_{\mathrm{g}}$, respectively. Although this DSJ has the form of a microstrip, the fringing fields are insignificant because $W \gg d_{\mathrm{i}}$. Therefore, the DSJ can be modelled as a parallelplate waveguide with an additional admittance term to represent the quasiparticle tunneling current.

The lumped-element equivalent circuit of the DSJ is shown in Fig. 1b [10]. In this model, the shunt capacitance $C$ and the series inductance $L$ per unit length are given by well-known equations for parallel-plate waveguides [11]:

$$
\begin{aligned}
C & =\frac{\varepsilon_{\mathrm{i}} W}{d_{\mathrm{i}}} \\
L & =\frac{\mu_{\mathrm{i}} d_{\mathrm{i}}}{W}
\end{aligned}
$$

where $\varepsilon_{\mathrm{i}}$ and $\mu_{\mathrm{i}}$ are the permitivity and permeability of the insulation barrier, respectively. Since the thickness of the insulation barrier $d_{\mathrm{i}}$ is not known precisely, the specific capacitance can be estimated using the empirical formula [12]:

$$
C_{\mathrm{s}}=\frac{0.3}{\ln \left(R_{\mathrm{n}} \cdot A_{\mathrm{J}}\right)}
$$

where $C_{\mathrm{s}}$ is in units $\mathrm{pF} / \mu \mathrm{m}^{2}, R_{\mathrm{n}}$ is the normal-state resistance in units $\Omega$, and $A_{\mathrm{J}}$ is the area of the junction in units $\mu \mathrm{m}^{2}$. By

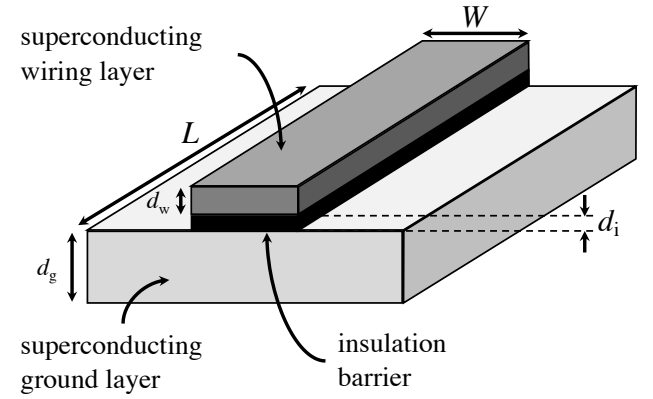

(a)

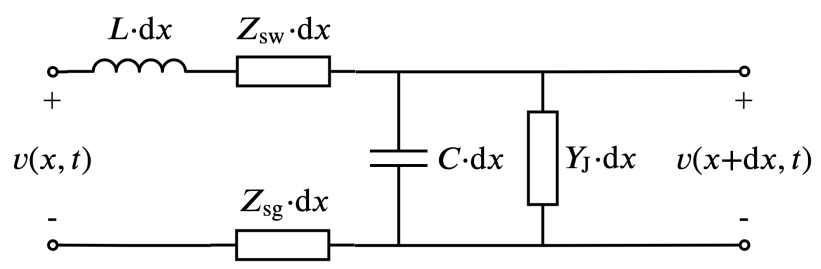

(b)

Figure 1. Modeling distributed SIS junctions (DSJs). A diagram of a DSJ is shown in (a) and the quasi-nonlinear transmission line model [10] is shown in (b). Here, $L$ and $C$ represent the inductance and capacitance per unit length, respectively; $Z_{\mathrm{sw}}$ and $Z_{\mathrm{sg}}$ represent the surface impedance of the superconducting wiring and ground layers, respectively; and $Y_{\mathrm{J}}$ represents the admittance of the quasiparticle tunneling current.

combining Eqns. 1 and 2, this empirical formula can also be adapted to estimate the specific inductance:

$$
L_{\mathrm{s}}=\frac{\mu_{\mathrm{i}} \varepsilon_{\mathrm{i}} \cdot \ln \left(R_{\mathrm{n}} \cdot A_{\mathrm{J}}\right)}{0.3}
$$

where $L_{\mathrm{s}}$ is in units $\mathrm{pH} / \mu \mathrm{m}^{2}$.

The surface impedance of the wiring and ground layers is represented in Fig. $1 \mathrm{~b}$ by $Z_{\mathrm{sw}}$ and $Z_{\mathrm{sg}}$, respectively. Since these layers are superconducting, Mattis-Bardeen theory [13] should be applied to estimate their values; however, for frequencies well below the gap frequency (below $\sim 350 \mathrm{GHz}$ for niobium), the surface impedance can be approximated by [14]:

$$
Z_{\mathrm{s}}=j \omega \mu \lambda_{\mathrm{L}} \cdot \operatorname{coth}\left(\frac{d}{\lambda_{\mathrm{L}}}\right)
$$

where $j=\sqrt{-1}$ is the imaginary unit, $\omega$ is the angular frequency, $\lambda_{\mathrm{L}}$ is the London penetration depth and $d$ is the thickness of the superconducting layer.

Finally, the admittance of the quasiparticle tunneling current is represented in Fig. $1 \mathrm{~b}$ by $Y_{\mathrm{J}}$. It is calculated by:

$$
Y_{\mathrm{J}}=\frac{I_{\omega}}{V_{\omega} \cdot A_{\mathrm{J}}}
$$

where $V_{\omega}$ is the $\mathrm{AC}$ voltage across the junction and $I_{\omega}$ is the consequent $\mathrm{AC}$ tunneling current. Both $V_{\omega}$ and $I_{\omega}$ are complex values. For $I_{\omega}$, the real and imaginary components are calculated by the large-signal tunneling equation ${ }^{1}[15]$ :

$$
\operatorname{Re}\left\{I_{\omega}\right\}=\sum_{m=-\infty}^{\infty} J_{m}(\alpha)\left[J_{m-p}(\alpha)+J_{m+p}(\alpha)\right]
$$

\footnotetext{
${ }^{1}$ Unlike [7]-[9], which use the small-signal admittance of the SIS junction, here we use the large-signal equations. We believe that the large-signal equations are more appropriate because the input signal is strong $(\alpha \sim 1)$.
} 


$$
\begin{array}{r}
\times \operatorname{Im}\left\{I_{\mathrm{R}}\left(V_{\mathrm{b}}+m V_{\mathrm{ph}}\right)\right\} \\
\operatorname{Im}\left\{I_{\omega}\right\}=\sum_{m=-\infty}^{\infty} J_{m}(\alpha)\left[J_{m-p}(\alpha)-J_{m+p}(\alpha)\right] \\
\times \operatorname{Re}\left\{I_{\mathrm{R}}\left(V_{\mathrm{b}}+m V_{\mathrm{ph}}\right)\right\}
\end{array}
$$

where $p$ is the harmonic number $(p=1$ for the fundamental frequency), $J_{m}$ is the $m^{\text {th }}$ order Bessel function of the first kind, $\alpha=\left|V_{\omega}\right| / V_{\mathrm{ph}}$ is the normalized $\mathrm{AC}$ voltage (hereafter referred to as the junction drive level), $V_{\mathrm{ph}}=\hbar \omega / e$ is the equivalent photon voltage, $e$ is the charge of an electron, $\hbar=h / 2 \pi$ is the reduced Planck constant, $I_{\mathrm{R}}$ is the response function of the SIS junction and $V_{\mathrm{b}}$ is the DC bias voltage. The response function is defined as:

$$
I_{\mathrm{R}}\left(V_{\mathrm{b}}\right) \equiv I_{\mathrm{kk}}^{0}\left(V_{\mathrm{b}}\right)+j I_{\mathrm{dc}}^{0}\left(V_{\mathrm{b}}\right)
$$

where $I_{\mathrm{dc}}^{0}$ is the DC tunneling current (with no AC signals present) and $I_{\mathrm{kk}}^{0}$ is the Kramers-Kronig transform of $I_{\mathrm{dc}}^{0}$ :

$$
I_{\mathrm{kk}}^{0}\left(V_{\mathrm{b}}\right)=\frac{1}{\pi} \mathcal{P} \int_{-\infty}^{\infty} \frac{I_{\mathrm{dc}}^{0}\left(V_{\mathrm{b}}^{\prime}\right)-V_{\mathrm{b}}^{\prime} / R_{\mathrm{n}}}{V_{\mathrm{b}}^{\prime}-V_{\mathrm{b}}} \mathrm{d} V_{\mathrm{b}}^{\prime} .
$$

Here, $\mathcal{P}$ represents the Cauchy principal value integral.

To simplify this model, the series components can be combined into a net impedance term $Z$ and the shunt components can be combined into a net admittance term $Y$ :

$$
\begin{aligned}
Z & =W^{-1} \cdot\left(j \omega L_{\mathrm{s}}+Z_{\mathrm{sw}}+Z_{\mathrm{sg}}\right) \\
Y & =W \cdot\left(j \omega C_{\mathrm{s}}+Y_{\mathrm{J}}\right) .
\end{aligned}
$$

We can then calculate the propagation constant $\gamma$ and the characteristic impedance $Z_{0}$ of the transmission line:

$$
\begin{aligned}
\gamma & =\sqrt{Z \cdot Y} \\
Z_{0} & =\sqrt{Z / Y} .
\end{aligned}
$$

If the DSJ is an open-circuit stub, such as the DSJ from [7][9], the input impedance can be calculated from:

$$
Z_{\text {in }}=Z_{0} \cdot \operatorname{coth}(\gamma L) \text {. }
$$

The current generated at the second harmonic $I_{\omega}^{\prime}$ is then calculated from Eqn. 6 with $p=2$. (The third and fourth harmonics can be calculated in a similar manner with $p=3$ and $p=4$, respectively.) Finally, the output power from the DSM is:

$$
P_{\text {out }}^{\prime}=\frac{1}{2}\left|I_{\omega}^{\prime}\right|^{2} \cdot \operatorname{Re}\left(Z_{\text {in }}\right) \text {. }
$$

\section{A. Simulated Results}

A simple DSJ model was generated for the purposes of this sub-section to illustrate the limitations of the quasi-nonlinear transmission line model. This DSJ has a width of $1 \mu \mathrm{m}$, a length of $20 \mu \mathrm{m}$ and it was treated as an open-circuit stub. The other properties of the DSJ are listed in Table I, including the properties of the niobium layers and the DC electrical properties. For the DC current-voltage relationship (i.e., the DC I-V curve), we used a polynomial model [16]:

$$
I_{\mathrm{dc}}^{0}\left(V_{\mathrm{b}}\right)=\frac{\left(V_{\mathrm{b}} / V_{\mathrm{gap}}\right)^{2 x+1}}{1+\left(V_{\mathrm{b}} / V_{\mathrm{gap}}\right)^{2 x}} \cdot \frac{V_{\mathrm{gap}}}{R_{\mathrm{n}}}
$$

Table I DSJ PROPERTIES ${ }^{\dagger}$

\begin{tabular}{lccc}
\hline Property & Variable & Value & Unit \\
\hline DSJ length & $L$ & 20 & $\mu \mathrm{m}$ \\
DSJ width & $W$ & 1 & $\mu \mathrm{m}$ \\
\hline Wiring layer thickness & $d_{\mathrm{w}}$ & 450 & $\mathrm{~nm}$ \\
Insulation barrier thickness & $d_{\mathrm{i}}$ & $\sim 1$ & $\mathrm{~nm}$ \\
Ground layer thickness & $d_{\mathrm{g}}$ & 250 & $\mathrm{~nm}$ \\
\hline London penetration depth & $\lambda_{\mathrm{L}}$ & 85 & $\mathrm{~nm}$ \\
Gap voltage & $V_{\mathrm{gap}}$ & 2.88 & $\mathrm{mV}$ \\
Normal-state resistance & $R_{\mathrm{n}}$ & 1.59 & $\Omega$ \\
\hline
\end{tabular}

These properties match those of the DSM device presented by Rashid et al. [7]-[9].

where $V_{\text {gap }}$ is the gap voltage, $R_{\mathrm{n}}$ is the normal-state resistance and the value $x$ controls the sharpness of the transition (set to $x=50$ for this example).

We simulated this DSJ using the quasi-nonlinear model (Eqns. 1-12) and the results are shown in Fig. 2. The top plot shows the propagation constant $\gamma=\alpha_{\gamma}+j \beta_{\gamma}$ multiplied by the length $L$ of the DSJ, which is the one-way propagation through the DSJ. (Note that the attenuation constant $\alpha_{\gamma}$ has a subscript to differentiate it from the junction drive level $\alpha$.) In the bottom plot, the input impedance $Z_{\text {in }}$ is plotted along with the characteristic impedance $Z_{0}$. The real component of the input impedance peaks whenever $\beta_{\gamma} \cdot L \approx n \pi$ for $n=1,2,3, \ldots$ In Fig. 2, this occurs at $179.0 \mathrm{GHz}, 351.3 \mathrm{GHz}, 509.8 \mathrm{GHz}$ and $644.9 \mathrm{GHz}$. There should also be a peak near $757.5 \mathrm{GHz}$, but above the gap frequency $(\sim 670 \mathrm{GHz})$, the attenuation is so high that the reflections from the open-circuit end are insignificant and so the input impedance approaches the value of the characteristic impedance. (To create a DSM that operates above $670 \mathrm{GHz}$, it would likely be necessary to use a material with a higher gap frequency, such as niobiumtitanium-nitride.)

So far, this quasi-nonlinear model has assumed that the junction drive level is constant across the entire DSJ. However, attenuation and reflections from the open-circuit end would normally cause the drive level to change with position, as shown in Fig. 3a. This is an issue because different drive levels should then cause the tunneling admittance $Y_{\mathrm{J}}$, the propagation constant $\gamma$ and the characteristic impedance $Z_{0}$ to change with position as well, especially for bias voltages close to $V_{\text {gap }}$ where the junction's admittance is very sensitive to $\alpha$. For example, in Fig. 3b, the one-way attenuation is $8.3 \mathrm{~dB}$ at a drive level of $\alpha=2$, but this quickly rises to $19.4 \mathrm{~dB}$ at $\alpha=0.5$. The phase constant and characteristic impedance also change significantly between $\alpha \sim 0.5$ and $\alpha \sim 2$, but the quasi-nonlinear model is unable to simulate how $Y_{J}, \gamma$ and $Z_{0}$ change with position along the DSJ. For that ability, we require a fully nonlinear transmission line model.

\section{Nonlinear DSJ TRANSMission Line Model}

The fully nonlinear DSJ transmission line model is presented in Fig. 4, with the model for the fundamental frequency in Fig. 4a and the model for the second harmonic 


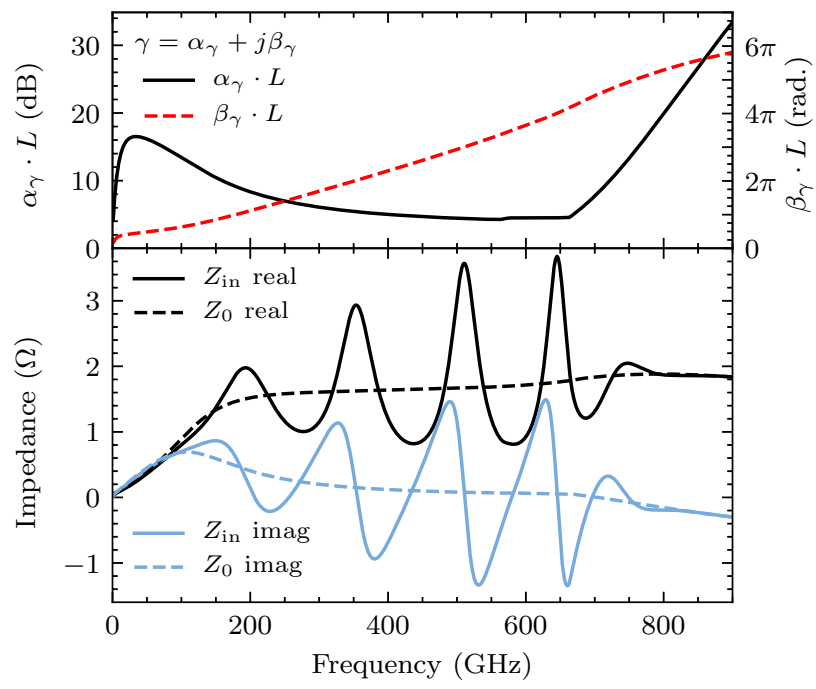

Figure 2. The simulated electrical properties from the quasi-nonlinear DSJ model. The top plot shows the one-way propagation, $\gamma \cdot L=\left(\alpha_{\gamma}+j \beta_{\gamma}\right) \cdot L$, and the bottom plot shows the input impedance $Z_{\text {in }}$ and characteristic impedance $Z_{0}$. For this simulation, the drive level was set to $\alpha=1$ and the bias voltage was set to $V_{\mathrm{b}}=V_{\mathrm{gap}}-V_{\mathrm{ph}} / 2$, which is the middle of the first photon step. The other properties of the DSJ are listed in Table I.

in Fig. 4b. The DSJ is now divided into $N$ segments where the segment length $\Delta x=L / N$ is set such that the drive level can be assumed to be constant over each unit length, similar to the analysis of distributed SIS mixers [17], [18]. (Using Fig. $3 \mathrm{a}$ as an example, if $\Delta x=L / 40$, the maximum error on $\alpha$ is always less than $5 \%$.) Unlike the quasi-nonlinear model, this new model allows $Y_{\mathrm{J}}$, the propagation constant and the characteristic impedance to change with position, depending on the magnitude of the $\mathrm{AC}$ voltage at that position. Furthermore, it includes the transmission line characteristics of the second harmonic, which were not included in the quasinonlinear model from Sec. II.

The circuit shown on the left-hand side of Fig. 4a represents the circuit within which the DSJ is embedded. If the embedding circuit is entirely linear, it can be reduced to a Thévenin equivalent circuit with voltage $V_{\mathrm{emb}}$ and impedance $Z_{\mathrm{emb}}$. At the second harmonic (Fig. $4 \mathrm{~b}$ ), there is no input power, so the embedding circuit is only represented by a load impedance $Z_{\mathrm{L}}^{\prime}$. (We denote the properties of the second harmonic using a superscript ${ }^{\prime}$.)

The transmission line model shown in Fig. $4 \mathrm{c}$ is then used to cascade the voltages and currents through the segments of Figs. $4 \mathrm{a}$ and $4 \mathrm{~b}$. This model is similar to the previous quasinonlinear transmission line model (Fig. 1b), except that the tunneling current is now able to change with position and the magnitude of the AC voltage. The tunneling current is also now represented by a voltage-dependant current source, instead of an admittance, which is necessary in order to

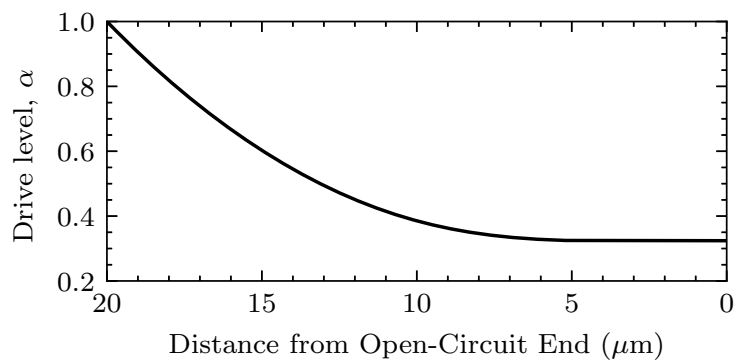

(a)

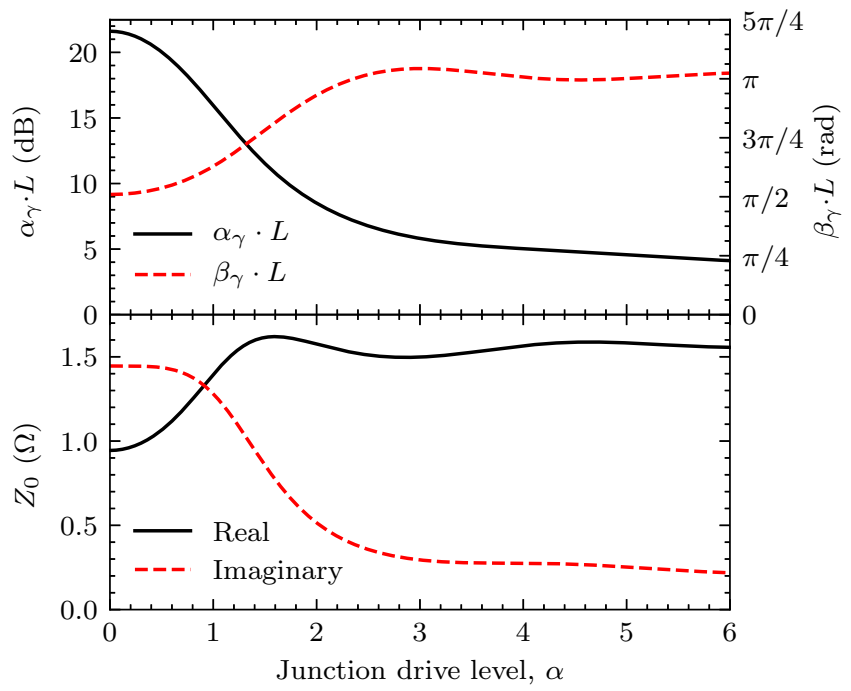

(b)

Figure 3. Investigating the limitations of the quasi-nonlinear DSJ model. For (a), we calculated $\gamma$ and $Z_{0}$ assuming $\alpha=1$ (Eqns. 1-10), and then we used these values to calculate $V_{\omega}$ and $\alpha$ versus position. As we can see, $\alpha \neq 1$. In (b), the electrical properties are plotted at different drive levels. For both plots, the bias voltage was set to $V_{\mathrm{b}}=V_{\text {gap }}$ and the frequency was set to $f=179.0 \mathrm{GHz}$ (the first peak in Fig. 2). Note that in (a), we would normally expect to see voltage maximums at both ends of the $\sim \lambda / 2$ open-circuit stub. However, due to the high attenuation seen in (b), we do not see this behavior since the reflections are too weak.

simulate the signal produced at the second harmonic ${ }^{2}$.

Using the transmission line model from Fig. $4 \mathrm{c}$, the voltages and currents cascade as:

$$
\left[\begin{array}{l}
V_{n+1} \\
I_{n+1}
\end{array}\right]=\left[\begin{array}{ll}
A & B \\
C & D
\end{array}\right]^{-1}\left[\begin{array}{l}
V_{n} \\
I_{n}
\end{array}\right]-\left[\begin{array}{c}
0 \\
I_{\omega ; n}\left(V_{n}, V_{n}^{\prime}\right)
\end{array}\right]
$$

where $n$ is the position index from Fig. 4a, the $A B C D$-matrix represents the linear components in Fig. $4 \mathrm{c}$, and $I_{\omega ; n}$ is the AC tunneling current in segment $n$ resulting from the voltages $V_{n}$ and $V_{n}^{\prime}$. Note that to calculate $I_{\omega ; n}$ using Eqn. 6, we have to replace $I_{\mathrm{R}}$ by the effective response function of a single segment of the DSJ model:

$$
I_{\mathrm{R}}^{s}\left(V_{\mathrm{b}}\right)=\frac{I_{\mathrm{R}}\left(V_{\mathrm{b}}\right)}{N}=\frac{I_{\mathrm{kk}}^{0}\left(V_{\mathrm{b}}\right)+j I_{\mathrm{dc}}^{0}\left(V_{\mathrm{b}}\right)}{N} .
$$

\footnotetext{
${ }^{2}$ As seen in Eqn. 6, the AC tunneling current is a function of the DC bias voltage $V_{\mathrm{b}}$ and the AC voltages $V_{n}$ and $V_{n}^{\prime}$. If power is dissipated along the DSJ (e.g., at the fundamental frequency), we can convert $I_{\omega}$ to an admittance value, as we did in Eqn. 5. If power is generated (e.g., at the second harmonic), $I_{\omega}^{\prime}$ should be modeled as a voltage-dependant current source (Fig. 4c). Here we decided to use voltage-dependent current sources for both harmonics, as given by Eqn. 6 .
} 


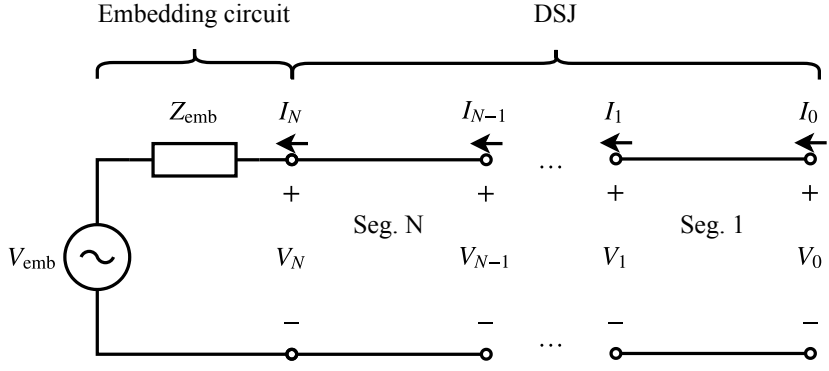

(a)

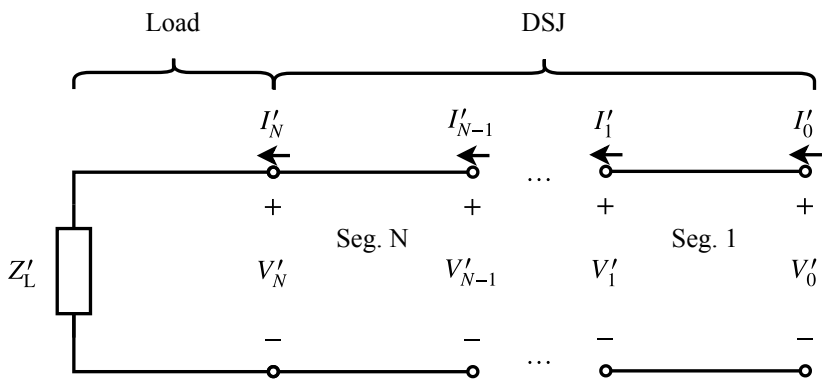

(b)

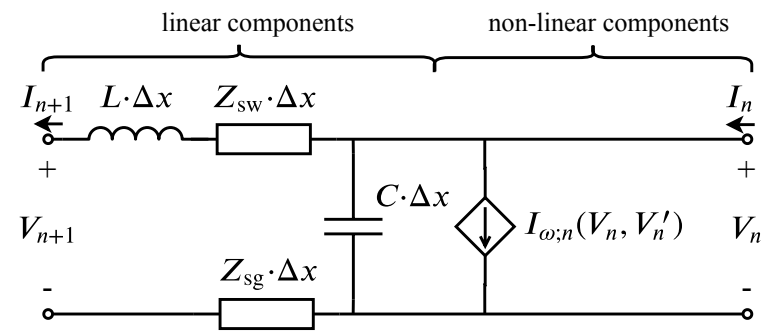

(c)

Figure 4. The nonlinear DSJ transmission line models for the fundamental frequency (a) and the second harmonic (b). In both models, the transmission line is divided into $N$ segments. Each segment in (a) uses the model shown in (c) to cascade the voltages and currents. There is an equivalent of (c) for the second harmonic, except that the first harmonic values are replaced with their second harmonic analogues (i.e., $V_{n} \rightarrow V_{n}^{\prime}, I_{n} \rightarrow I_{n}^{\prime}, I_{\omega ; n} \rightarrow I_{\omega ; n}^{\prime}$, etc.).

In other words, since $I_{\mathrm{R}}$ is proportional to $I_{\mathrm{dc}}^{0}$, which is itself proportional to the area of the junction, we have to divide $I_{\mathrm{R}}$ by $N$ to solve for the single-segment response function $I_{\mathrm{R}}^{s}$.

In Eqn. 14, the complex coefficients of the $A B C D$-matrix are calculated by [11]:

$$
\begin{aligned}
& A=\cosh (\gamma \cdot \Delta x) \\
& B=\sinh (\gamma \cdot \Delta x) \cdot Z_{0} \\
& C=\sinh (\gamma \cdot \Delta x) / Z_{0} \\
& D=\cosh (\gamma \cdot \Delta x) .
\end{aligned}
$$

The propagation constant $\gamma$ and characteristic impedance $Z_{0}$ are again calculated by Eqn. 10, except that the tunneling current is no longer included in the admittance term $Y$ (Eqn. 9b). Instead, the admittance term is simply:

$$
Y=W \cdot j \omega C_{\mathrm{s}} .
$$

For Eqns. 14-17, there is also an identical set of equations for the second harmonic, except that the first harmonic values are replaced with their second harmonic analogues, i.e., $V_{n} \rightarrow V_{n}^{\prime}$, $I_{n} \rightarrow I_{n}^{\prime}, I_{\omega ; n} \rightarrow I_{\omega ; n}^{\prime}, \gamma \rightarrow \gamma^{\prime}$, and $Z_{0} \rightarrow Z_{0}^{\prime}$.
With the nonlinear DSJ model in place, it is then necessary to solve for the voltages $V_{0}$ and $V_{0}^{\prime}$ that satisfy the embedding circuits for both harmonics simultaneously. (Recall that $V_{0}$ represents the voltage at position index 0 and not the DC bias voltage, which is represented by $V_{\mathrm{b}}$.) To accomplish this, we began by assuming initial values for $V_{0}$ and $V_{0}^{\prime}$. ( $I_{0}$ and $I_{0}^{\prime}$ are equal to zero at the open-circuit end of the DSJ.) We found that initial values of $V_{0}=V_{\mathrm{emb}}$ and $V_{0}^{\prime}=V_{\mathrm{emb}} \div 10$ were typically adequate. These values were then cascaded to the opposite end of the DSJ using Eqn. 14. For the first and second harmonics, respectively, the resulting voltage errors at position index $N$ are:

$$
\begin{aligned}
& \Delta=I_{N} \cdot Z_{\mathrm{emb}}-V_{N}-V_{\mathrm{emb}} \\
& \Delta^{\prime}=I_{N}^{\prime} \cdot Z_{\mathrm{L}}^{\prime}-V_{N}^{\prime} .
\end{aligned}
$$

The goal is then to find $V_{0}$ and $V_{0}^{\prime}$ such that $\Delta$ and $\Delta^{\prime}$ are equal to zero. This is challenging because the equations are highly nonlinear functions of $V_{0}$ and $V_{0}^{\prime}$, and the variables are complex values with both real and imaginary components.

To find a solution to Eqn. 18, we used the Newton-Raphson method, which is an iterative root-finding algorithm, similar to the harmonic balance procedure presented in [19]. Through each iteration, the estimated $\mathrm{AC}$ voltage is updated by:

$$
\mathbf{V}_{0}^{k+1}=\mathbf{V}_{0}^{k}-\left[J\left(\mathbf{V}_{0}^{k}\right)\right]^{-1} \cdot \Delta\left(\mathbf{V}_{0}^{k}\right)
$$

where $k$ represents the iteration number and the bold face notation represents column vectors:

$$
\mathbf{V}_{0}=\left[\begin{array}{l}
\operatorname{Re} V_{0} \\
\operatorname{Im} V_{0} \\
\operatorname{Re} V_{0}^{\prime} \\
\operatorname{Im} V_{0}^{\prime}
\end{array}\right] ; \quad \boldsymbol{\Delta}=\left[\begin{array}{l}
\operatorname{Re} \Delta \\
\operatorname{Im} \Delta \\
\operatorname{Re} \Delta^{\prime} \\
\operatorname{Im} \Delta^{\prime}
\end{array}\right] .
$$

$J\left(\mathbf{V}_{0}\right)$ is then the Jacobian of Eqn. 18, which represents the sensitivity of the error $\Delta$ to small perturbations in $\mathbf{V}_{0}$. For a DSJ transmission line with two harmonics, the Jacobian will be a $4 \times 4$ matrix with values:

$$
J\left(\mathbf{V}_{0}\right)=\left[\begin{array}{llll}
\frac{\partial \operatorname{Re} \Delta}{\partial \operatorname{Re} V_{0}} & \frac{\partial \operatorname{Re} \Delta}{\partial \operatorname{Im} V_{0}} & \frac{\partial \operatorname{Re} \Delta}{\partial \operatorname{Re} V_{0}^{\prime}} & \frac{\partial \operatorname{Re} \Delta}{\partial \operatorname{Im} V_{0}^{\prime}} \\
\frac{\partial \operatorname{Im} \Delta}{\partial \operatorname{Re} V_{0}} & \frac{\partial \operatorname{Im} \Delta}{\partial \operatorname{Im} V_{0}} & \frac{\partial \operatorname{Im} \Delta}{\partial \operatorname{Re} V_{0}^{\prime}} & \frac{\partial \operatorname{Im} \Delta}{\partial \operatorname{Im} V_{0}^{\prime}} \\
\frac{\partial \operatorname{Re} \Delta^{\prime}}{\partial \operatorname{Re} V_{0}} & \frac{\partial \operatorname{Re} \Delta^{\prime}}{\partial \operatorname{Im} V_{0}} & \frac{\partial \operatorname{Re} \Delta^{\prime}}{\partial \operatorname{Re} V_{0}^{\prime}} & \frac{\partial \operatorname{Re} \Delta^{\prime}}{\partial \operatorname{Im} V_{0}^{\prime}} \\
\frac{\partial \operatorname{Im} \Delta^{\prime}}{\partial \operatorname{Re} V_{0}} & \frac{\partial \operatorname{Im} \Delta^{\prime}}{\partial \operatorname{Im} V_{0}} & \frac{\partial \operatorname{Im} \Delta^{\prime}}{\partial \operatorname{Re} V_{0}^{\prime}} & \frac{\partial \operatorname{Im} \Delta^{\prime}}{\partial \operatorname{Im} V_{0}^{\prime}}
\end{array}\right] .
$$

In our experience, this method typically requires 5-15 iterations to converge on a solution, depending on the embedding circuit, the response function and the bias voltage.

Once the simulation is complete, the power delivered to the load at the second harmonic is calculated by:

$$
P_{\text {out }}^{\prime}=\frac{\left|V_{N}^{\prime}\right|^{2}}{2 \cdot \operatorname{Re}\left\{Z_{\mathrm{L}}^{\prime}\right\}} \text {. }
$$

This value is then used to calculate the efficiency of the DSM:

$$
\eta=\frac{P_{\mathrm{out}}^{\prime}}{P_{\mathrm{emb}}}
$$


where $P_{\mathrm{emb}}$ is the available power from the embedding circuit:

$$
P_{\mathrm{emb}}=\frac{\left|V_{\mathrm{emb}}\right|^{2}}{8 \cdot \operatorname{Re}\left\{Z_{\mathrm{emb}}\right\}} .
$$

The goal of any DSM device should be to maximize the efficiency $\eta$ and the output power $P_{\text {out }}^{\prime}$.

\section{Comparing the New Nonlinear DSJ Model to EXPERIMENTAL DATA}

We recently reported a new DSM device that uses two $1 \mu \mathrm{m} \times 20 \mu \mathrm{m}$ DSJs mounted in parallel, with each DSJ added to the planar circuit as an open-circuit stub [7]-[9]. Bandpass filters were placed on either side of the DSJs to set the direction of the output signal and to prevent leakage between the input and output waveguides. The electrical properties and the geometry of the DSJs are listed in Table I and a diagram of the planar circuit can be found in [7].

When we tested this device, the input signal was injected at $93 \mathrm{GHz}$ and this produced an output signal at $186 \mathrm{GHz}$, which is double the input frequency. During the experiment, we recorded the DC tunneling current, with and without the input signal applied, and we measured the output power using a separate SIS mixer chip. We then de-embedded the measured power by estimating the loss between the DSM and the SIS mixer. In addition to the loss estimated in [7]-[9], in this paper we have also included $1 \mathrm{~dB}$ of loss from the waveguide probes (due to a scaling error in the experimental device), $1.4 \mathrm{~dB}$ of loss due to the conversion gain of the SIS mixer [20] and $3.3 \mathrm{~dB}$ of loss due to the impedance mismatch between the SIS device and the IF measurement circuit.

Previously, Rashid et al. [7]-[9] compared the experimental data from this DSM device to the quasi-nonlinear model from Sec. II. In this section, we now compare the experimental data to the new fully nonlinear model from Sec. III. First, we used the experimental DC currents to estimate the properties of the embedding circuit (Sec. IV-A), and then we simulated the AC signal at the fundamental frequency (Sec. IV-B) and the output power at the second harmonic (Sec. IV-C).

For the simulations in this section, we used the QMix software package ${ }^{3}$ to calculate the quasiparticle tunneling currents [21]-[23], instead of Eqn. 6. QMix uses multitone spectral domain analysis [24]-[26], which makes the software ideal for simulating higher-order harmonics, such as those present within DSJ transmission lines. It also has built-in utilities for importing and filtering the experimental DC I-V curve, calculating the Kramers-Kronig transform and interpolating the response function. Furthermore, the QMix code has extensive unit tests, which reduces the likelihood of errors in our simulations. In brief, we could have used Eqn. 6 alone, but we chose to use QMix since it is a powerful software package that facilitates working with experimental data.

\section{A. Recovering the Embedding Circuit}

To begin, we recovered the properties of the embedding circuit by analyzing the experimental DC tunneling currents,

${ }^{3}$ Online: https://github.com/garrettj403/QMix similar to what is normally done with SIS mixers [27], [28]. We started by estimating a voltage at position index 0 and cascading this value across the DSJ to find all of the voltages $V_{n}$ and currents $I_{n}$. Using these values, we then calculated the DC tunneling current in each segment $n$ [15]:

$$
I_{\mathrm{dc} ; \mathrm{n}}\left(V_{\mathrm{b}}\right)=\sum_{m=-\infty}^{\infty} J_{m}^{2}\left(\alpha_{n}\right) \cdot \operatorname{Im}\left\{I_{\mathrm{R}}^{s}\left(V_{\mathrm{b}}\right)\right\}
$$

where $\alpha_{n}=\left|V_{n}\right| / V_{\mathrm{ph}}$ is the drive level of segment $n$, and $I_{\mathrm{R}}^{s}\left(V_{\mathrm{b}}\right)$ is the single-segment response function (Eqn. 15). The total DC tunneling current through the DSJ is then:

$$
I_{\mathrm{dc}}\left(V_{\mathrm{b}}\right)=\sum_{n=0}^{N-1} I_{\mathrm{dc} ; \mathrm{n}}\left(V_{\mathrm{b}}\right) \text {. }
$$

By comparing this value to the experimental DC current, we revised our $V_{0}$ estimate through several iterations to recover $V_{n}$ and $I_{n}$ at each bias voltage. The embedding circuit was then estimated from the $V_{N}$ and $I_{N}$ values using a least squares fitting [28]. With SIS mixers, this technique is normally performed over the first photon step, but here we used the first 5 photon steps to improve the accuracy of the estimation, i.e., from $V_{\mathrm{b}} \sim 1 \mathrm{mV}$ to $V_{\mathrm{b}} \sim 2.8 \mathrm{mV}$. For this data set, the estimated embedding voltage and impedance were $V_{\mathrm{emb}}=2.95 \mathrm{mV}$ and $Z_{\mathrm{emb}}=1.20+j 1.36 \Omega$, respectively, giving the embedding circuit an estimated available power of $P_{\mathrm{emb}}=907 \mathrm{nW}$.

Using this embedding circuit, we then simulated the DC tunneling current, as shown in Fig. 5. Below the gap voltage, the simulated current is very close to the experimental value with an RMS error of $0.7 \mu \mathrm{A}$ between $0 \mathrm{mV}$ and $2.8 \mathrm{mV}$. Above $\sim 2.6 \mathrm{mV}$, heating effects likely lower the effective gap voltage, resulting in a leftward shift in the experimental DC tunneling current. For comparison, we have also included the quasi-nonlinear model from Sec. II in Fig. 5. As we can see, the new nonlinear model has a much better fit to the experimental data, suggesting that it provides a more accurate description of the DSJ's behavior.

\section{B. AC Properties at the Fundamental Frequency}

Next, we simulated the AC signal at the fundamental frequency. In Fig. 6, the drive level and AC power are plotted as a function of position. The AC power at position index $n$ was calculated by:

$$
P_{n}=\frac{1}{2} \operatorname{Re}\left\{V_{n} \cdot I_{n}^{*}\right\}
$$

where $I_{n}^{*}$ represents the complex conjugate of $I_{n}$. As expected, the $\mathrm{AC}$ power decreases monotonically with distance along the DSJ. In contrast, the drive level both increases and decreases as a function of position and bias voltage. Across all positions and voltages, the maximum simulated drive level is $\alpha=6.1$ at $\left(n, V_{\mathrm{b}}\right)=(0,0.0 \mathrm{mV})$ and the minimum is $\alpha=0.1$ at $\left(n, V_{\mathrm{b}}\right)=(37,0.1 \mathrm{mV})$. Recall that the quasi-nonlinear model did not allow $Y_{\mathrm{J}}$ to change with $\alpha$ or position, which limited the accuracy of the simulations. 


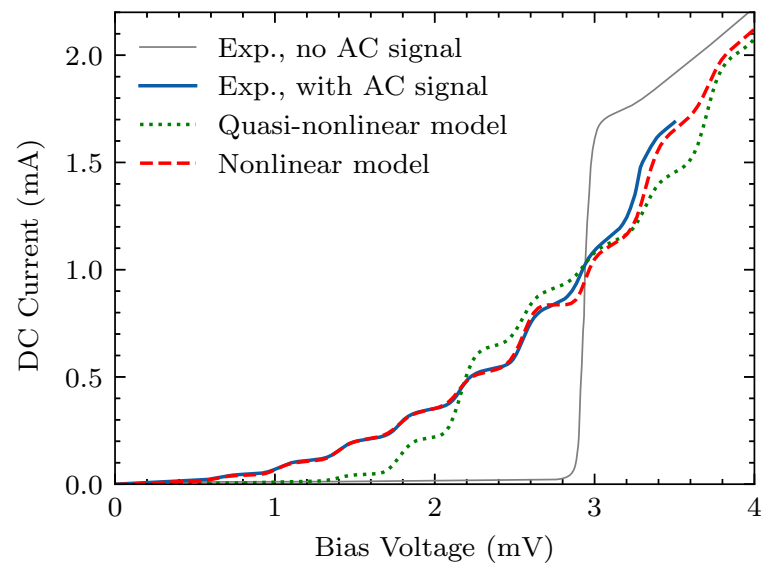

Figure 5. The DC tunneling current from the nonlinear model compared to experimental data. For the simulation, the embedding voltage was $V_{\mathrm{emb}}=2.95 \mathrm{mV}$ and the embedding impedance was $Z_{\mathrm{emb}}=1.20+j 1.36 \Omega$. The frequency of the fundamental harmonic was $93 \mathrm{GHz}$. The DC tunneling current from the quasi-nonlinear model is also included (assuming $\alpha=3$ ).

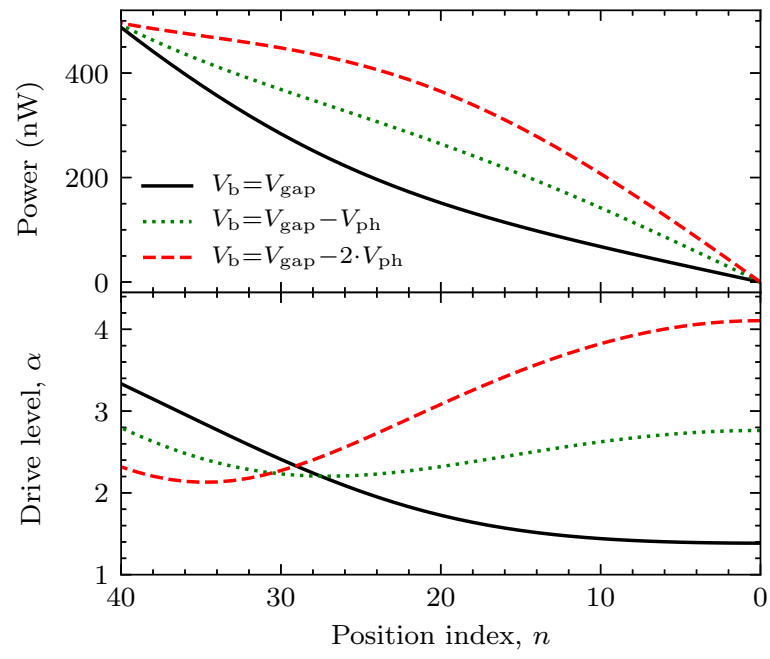

Figure 6. The simulated AC power (top) and drive level (bottom) at the fundamental frequency $(93 \mathrm{GHz})$. The position index $n$ corresponds to the indices shown in Fig. 4. The total number of segments was $N=40$.

\section{Output Power at the Second Harmonic}

Finally, we simulated the output power from the DSM device at the second harmonic. In Fig. 7, the simulated power is compared to the experimental data and the quasi-nonlinear model from Sec. II. For the nonlinear DSJ simulation, we used the embedding circuit from Sec. IV-A, but the precise value of the load impedance $Z_{\mathrm{L}}^{\prime}$ at the second harmonic was unknown. In our previous work [7], we estimated that the value of $Z_{\mathrm{L}}^{\prime}$ should be approximately $2.3 \Omega$; therefore, for our simulation, we swept the magnitude of $Z_{\mathrm{L}}^{\prime}$ from $1 \Omega$ to $4 \Omega$ and the phase from $-90^{\circ}$ to $+90^{\circ}$. From these values, the simulated output power with $Z_{\mathrm{L}}^{\prime}=1.6-j 1.6 \Omega$ was found to provide the best match to the experimental data. This value is equivalent to $Z_{\mathrm{L}}^{\prime}=2.3 \angle 45^{\circ} \Omega$, which is consistent with our previous estimation. It could be possible to improve the fit to the experimental data by using more accurate values for $Z_{\mathrm{emb}}$ and $Z_{\mathrm{L}}^{\prime}$, but it is challenging to recover these impedances from

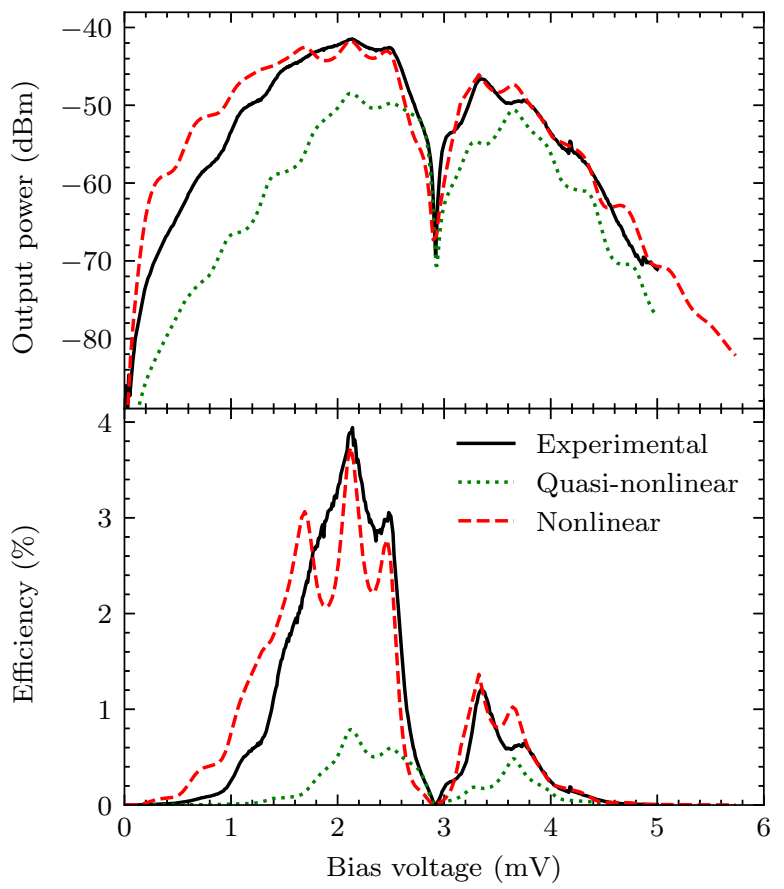

Figure 7. The output power (top) and conversion efficiency (bottom) of the DSM device. For the nonlinear model, we used the embedding circuit from Sec. IV-A and a load impedance of $Z_{\mathrm{L}}^{\prime}=1.6-j 1.6 \Omega$. We have also included the results from the quasi-nonlinear model for comparison.

the experimental data.

Despite the uncertainty in $Z_{\mathrm{emb}}$ and $Z_{\mathrm{L}}^{\prime}$, the agreement between the nonlinear DSJ model and the experimental results is very good, with an excellent fit above $\sim 1.6 \mathrm{mV}$. The new model also provides a much better fit than the quasinonlinear model, which estimates a significantly lower output power (Fig. 7). Overall, these results demonstrate that the new nonlinear model is able to accurately simulate the experimental behavior of the DSJ, suggesting that it can be used to design new DSM devices and optimize their performance.

\section{Optimizing a New DSM DeVice}

In the nonlinear DSJ model, there are five degrees of freedom, not including the properties of the DSJ itself. These parameters are the bias voltage $V_{\mathrm{b}}$, the embedding voltage $V_{\text {emb }}$, the embedding impedance $Z_{\text {emb }}$, the load impedance $Z_{\mathrm{L}}^{\prime}$ and the frequency of the fundamental tone $f$. The impedance values can also split into their real and imaginary components, which would result in two additional degrees of freedom. Optimizing all of these values is computationally expensive; however, we made an initial attempt by constraining several of the free parameters.

To begin, we set the frequency to $100 \mathrm{GHz}$ and the bias voltage to $V_{\mathrm{b}}=V_{\mathrm{gap}}-2 \cdot V_{\mathrm{ph}}$, which is the location of the maximum output power in the experimental data (Fig. 7). We then simulated the DSJ assuming a strong input signal $\left(\left|V_{n}\right| \sim 3 \cdot V_{\mathrm{ph}}\right)$ and found that the length of the DSJ is equal to $\lambda / 2$ when $L=35 \mu \mathrm{m}$, where $\lambda$ is the guided wavelength. For this length, the input impedance of the DSJ is $Z_{\text {in }}=7.56+j 0.59 \Omega$. By taking the complex conjugate of this value (to maximize the 


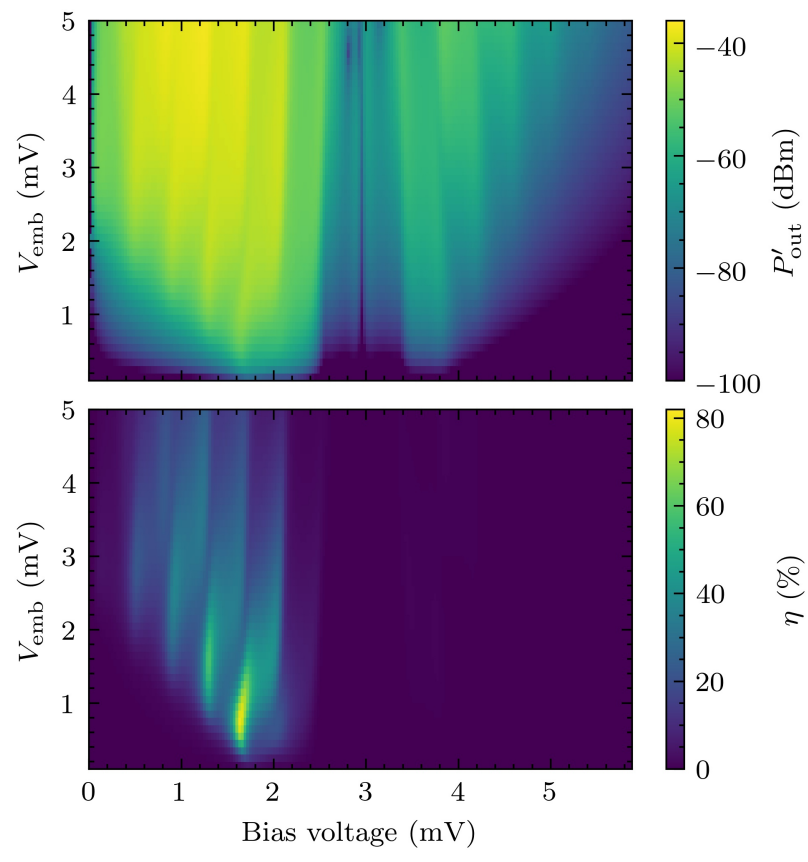

Figure 8. The simulated output power (top) and efficiency (bottom) of the optimized DSM device. For this simulation, the frequency was $100 \mathrm{GHz}$, the length was $35 \mu \mathrm{m}$, the embedding voltage was $Z_{\mathrm{emb}}=7.56-j 0.59 \Omega$ and the load impedance was $Z_{\mathrm{L}}^{\prime}=5+j 20 \Omega$.

power delivered to the DSJ), we estimated an optimal embedding impedance of $Z_{\mathrm{emb}}=7.56-j 0.59 \Omega$. Finally, we swept the real and imaginary components of the load impedance $Z_{\mathrm{L}}^{\prime}$ and simulated the output power from each value. We found that inductive $Z_{\mathrm{L}}^{\prime}$ values provide the highest output powers.

As seen in Fig. 8, with $Z_{\mathrm{L}}^{\prime}=5+j 20 \Omega$, the optimized DSJ simulation provides an output power of $-37 \mathrm{dBm}$ at $\left(V_{\mathrm{b}}, V_{\mathrm{emb}}\right)=(1.24,5.0) \mathrm{mV}$ and an efficiency of $80 \%$ at $\left(V_{\mathrm{b}}, V_{\mathrm{emb}}\right)=(1.62,0.7) \mathrm{mV}$. Since SIS junctions require approximately $-40 \mathrm{dBm}$ of LO power, this power level would be enough to pump an SIS mixer (if the signal were injected into the mixer through an RF hybrid). The maximum conversion efficiency shown in Fig. 8 is lower than the value predicted by the quasi-nonlinear DSJ model ( $\eta \sim 95 \%$ [7]), but it is much higher than the efficiency of modern Schottky diode frequency multipliers ${ }^{4}(\eta \sim 30 \%$ at $200 \mathrm{GHz}$ [29]). We suspect that the efficiency can be extended beyond $80 \%$ by further optimizing the DSJ model. For example, in future work we could simulate a wider range of parameters or use a better optimization algorithm. We could also use a DSJ with a higher current density, which would allow us to achieve a higher input impedance and likely a better match to the embedding circuit.

The proposed DSJ is $75 \%$ larger than the DSJ presented by Rashid et al. [7]-[9]. Based on our experience, it should be feasible to fabricate a junction of this size. The highly inductive embedding impedance at the second harmonic could be more difficult to achieve; however, it should also be

\footnotetext{
${ }^{4}$ Recall that the motivation for DSM devices is high conversion efficiency at frequencies above $\sim 100 \mathrm{GHz}$, mostly for $\mathrm{LO}$ chains in astronomical receivers Schottky diodes still offer many advantages including the ability to operate at room temperature with much higher signal powers.
}

attainable since it is only required over a limited frequency range.

\section{CONCLUSION}

In this paper, we presented a new nonlinear transmission line model for simulating distributed SIS frequency multipliers. Unlike previous models, this new model captures how the tunneling current changes with position and $\mathrm{AC}$ power, allowing for nonlinear propagation parameters and more accurate simulations. We compared the new nonlinear model to experimental data and found very good agreement. Both the simulated DC tunneling current and the output power at the second harmonic closely matched the experimental data; although, there was some uncertainty in the experimental values. Based on this success, we simulated the optimal embedding circuit to maximize the conversion efficiency and output power at the second-harmonic. According to these simulations, an optimized SIS frequency doubler should be able to provide an efficiency of $80 \%$ and an output power of $-37 \mathrm{dBm}$, which is enough to drive an SIS mixer. We suspect that the performance could be further optimized in the future, allowing for SIS frequency multipliers with even higher conversion efficiencies. Overall, this work will help to develop efficient local-oscillator sources and support new astronomical receivers at millimeter and submillimeter wavelengths, including SIS receivers that operate above $1 \mathrm{THz}$ and large-scale focal plane arrays. In future work, we hope to fabricate the optimized DSM device and continue to validate the nonlinear DSJ model with additional experimental data.

\section{REFERENCES}

[1] V. Belitsky, M. Bylund, V. Desmaris, A. Ermakov, S.-E. Ferm, M. Fredrixon, S. Krause, I. Lapkin, D. Meledin, A. Pavolotsky, H. Rashid, S. Shafiee, M. Strandberg, E. Sundin, P. Y. Aghdam, R. Hesper, J. Barkhof, M. E. Bekema, J. Adema, R. de Haan, A. Koops, W. Boland, P. Yagoubov, G. Marconi, G. Siringo, E. Humphreys, G. H. Tan, R. Laing, L. Testi, T. Mroczkowski, W. Wild, K. S. Saini, and E. Bryerton, "ALMA Band 5 receiver cartridge," Astron. Astrophys., vol. 611, no. 20, p. A98, Mar. 2018, doi: 10.1051/0004-6361/201731883.

[2] A. M. Baryshev, R. Hesper, F. P. Mena, T. M. Klapwijk, T. A. van Kempen, M. R. Hogerheijde, B. D. Jackson, J. Adema, G. J. Gerlofsma, M. E. Bekema, J. Barkhof, L. H. R. de Haan-Stijkel, M. van den Bemt, A. Koops, K. Keizer, C. Pieters, J. Koops van het Jagt, H. H. A. Schaeffer, T. Zijlstra, M. Kroug, C. F. J. Lodewijk, K. Wielinga, W. Boland, M. W. M. de Graauw, E. F. van Dishoeck, H. Jager, and W. Wild, "The ALMA Band 9 receiver," Astron. Astrophys., vol. 577, no. A129, p. 12, May 2015, doi: 10.1051/0004-6361/201425529.

[3] A. R. Kerr, S.-K. Pan, S. M. X. Claude, P. Dindo, A. W. Lichtenberger, J. E. Effland, and E. F. Lauria, "Development of the ALMA Band-3 and Band-6 Sideband-Separating SIS Mixers," IEEE Trans. $\mathrm{THz}$ Sci. Technol., vol. 4, no. 2, pp. 201-212, Mar. 2014, doi: 10.1109/TTHZ.2014.2302537.

[4] A. Remijan, A. Biggs, P. A. Cortes, B. Dent, J. Di Francesco, E. Fomalont, A. Hales, S. Kameno, B. Mason, N. Philips, K. Saini, F. Stoehr, B. Vila Vilaro, and E. Villard, "ALMA Technical Handbook," ALMA Doc. 7.3, ver. 1.0, Tech. Rep., Mar. 2019.

[5] Y. Uzawa, M. Kroug, T. Kojima, M. Takeda, M. Candotti, Y. Fujii, K. Kaneko, W. Shan, T. Noguchi, and Z. Wang, "A sensitive ALMA Band 10 SIS receiver engineering model," Supercond. Sci. Technol., vol. 22, no. 11, p. 114002, Nov. 2009, doi: 10.1088/09532048/22/11/114002.

[6] B. Billade, A. Pavolotsky, and V. Belitsky, "Experimental Study of Frequency Multiplication in a Distributed Array of SIS Junctions," IEEE Trans. THz Sci. Technol., vol. 4, no. 2, pp. 254-259, Mar. 2014, doi: 10.1109/TTHZ.2014.2304138. 
[7] H. Rashid, S. Krause, D. Meledin, V. Desmaris, A. Pavolotsky, and V. Belitsky, "Frequency Multiplier Based on Distributed Superconducting Tunnel Junctions: Theory, Design, and Characterization," IEEE Trans. THz Sci. Technol., vol. 6, no. 5, pp. 724-736, Sep. 2016, doi: 10.1109/TTHZ.2016.2583201.

[8] H. Rashid, V. Desmaris, A. Pavolotsky, and V. Belitsky, "THz Frequency Up-Conversion using Superconducting Tunnel Junction," IEEE Microw. Wirel. Compon. Lett., vol. 26, no. 10, pp. 831-833, Oct. 2016, doi: 10.1109/LMWC.2016.2605453.

[9] _ - "Harmonic and reactive behavior of the quasiparticle tunnel current in SIS junctions," AIP Adv., vol. 6, no. 4, p. 045109, Apr. 2016, doi: 10.1063/1.4947133.

[10] V. Y. Belitsky and E. L. Kollberg, "Superconductor-insulatorsuperconductor tunnel strip line: Features and applications," J. Appl. Phys., vol. 80, no. 8, pp. 4741-4748, Oct. 1996, doi: 10.1063/1.363411.

[11] D. M. Pozar, Microwave engineering, 3rd ed. Hoboken, NJ: John Wiley \& Sons, Inc., 2005.

[12] V. Y. Belitsky, S. W. Jacobsson, S. A. Kovtonjuk, E. L. Kollberg, and A. B. Ermakov, "100 GHz mixer vertically integrated (stacked) SIS junction array," J. Infrared Millim. Terahertz Waves, vol. 14, no. 5, pp. 949-957, May 1993, doi: 10.1007/BF02084571.

[13] D. C. Mattis and J. Bardeen, "Theory of the Anomalous Skin Effect in Normal and Superconducting Metals," Phys. Rev., vol. 111, no. 2, pp. 412-417, Jul. 1958, doi: 10.1103/PhysRev.111.412.

[14] A. R. Kerr, "Surface Impedance of Superconductors and Normal Conductors in EM Simulators," National Radio Astronomy Observatory, Green Bank, West Virginia, Tech. Rep. 302, Feb. 1996.

[15] J. R. Tucker and M. J. Feldman, "Quantum detection at millimeter wavelengths," Rev. Mod. Phys., vol. 57, no. 4, pp. 1055-1113, Oct. 1985, doi: 10.1103/RevModPhys.57.1055.

[16] P. Kennedy, "Superconducting Devices for Millimeter Wavelength Astronomy," DPhil thesis, University of Cambridge, 1999.

[17] C.-Y. E. Tong, L. Chen, and R. Blundell, "Theory of distributed mixing and amplification in a superconducting quasi-particle nonlinear transmission line," IEEE Trans. Microw. Theory Techn., vol. 45, no. 7, pp. 1086-1092, Jul. 1997, doi: 10.1109/22.598445.

[18] C. Tong, R. Blundell, B. Bumble, J. Stern, and H. LeDuc, "Distributed quasiparticle mixing in a non-linear transmission line at sub-millimeter wavelengths," IEEE Trans. Appl. Supercond., vol. 7, no. 2, pp. 35973600, Jun. 1997, doi: 10.1109/77.622181.

[19] S. Withington and P. Kennedy, "Numerical procedure for simulating the large-signal quantum behaviour of superconducting tunnel-junction circuits," IEE Proceedings G Circuits, Devices and Systems, vol. 138, no. 1, pp. 70-76, Feb. 1991, doi: 10.1049/ip-g-2.1991.0014.

[20] B. Billade, A. Pavolotsky, and V. Belitsky, "An SIS Mixer with $2 h f / k$ DSB Noise Temperature at $163-211 \mathrm{GHz}$ Band," IEEE Trans. THz Sci. Technol., vol. 3, no. 4, pp. 416-421, Jul. 2013, doi: 10.1109/TTHZ.2013.2255734.

[21] J. D. Garrett and G. Yassin, "QMix: A Python package for simulating the quasiparticle tunneling currents in SIS junctions," J. Open Source Softw., vol. 4, no. 35, p. 1231, Mar. 2019, doi: 10.21105/joss.01231.

[22] J. D. Garrett, B. K. Tan, F. Boussaha, C. Chaumont, and G. Yassin, "Simulating the Behavior of a 230-GHz SIS Mixer Using Multitone Spectral Domain Analysis," IEEE Trans. THz Sci. Technol., vol. 9, no. 6, pp. 540-548, Nov. 2019, doi: 10.1109/TTHZ.2019.2938993.

[23] J. D. Garrett, "A $230 \mathrm{GHz}$ Focal Plane Array Using a Wide IF Bandwidth SIS Receiver," DPhil thesis, University of Oxford, 2018.

[24] P. Kittara, "The Development of a $700 \mathrm{GHz}$ SIS Mixer with Nb Finline Devices: Nonlinear Mixer Theory, Design Techniques and Experimental Investigation," DPhil thesis, University of Cambridge, 2002.

[25] S. Withington, P. Kittara, and G. Yassin, "Multitone quantum simulations of saturating tunnel junction mixers," J. Appl. Phys., vol. 93, no. 12, pp. 9812-9822, Jun. 2003, doi: 10.1063/1.1576515.

[26] P. Kittara, S. Withington, and G. Yassin, "Theoretical and numerical analysis of very high harmonic superconducting tunnel junction mixers," J. Appl. Phys., vol. 101, no. 2, p. 024508, Jan. 2007, doi: $10.1063 / 1.2424407$

[27] A. Skalare, "Determining embedding circuit parameters from DC measurements on quasiparticle mixers," J. Infrared Millim. Terahertz Waves, vol. 10, no. 11, pp. 1339-1353, Nov. 1989, doi: 10.1007/BF01010125.

[28] S. Withington, K. Isaak, S. Kovtonyuk, R. Panhuyzen, and T. Klapwijk, "Direct detection at submillimetre wavelengths using superconducting tunnel junctions," Infrared Phys. Technol., vol. 36, no. 7, pp. 1059-1075, Dec. 1995, doi: 10.1016/1350-4495(95)00058-5.

[29] I. Mehdi, J. V. Siles, C. Lee, and E. Schlecht, "THz Diode Technology: Status, Prospects, and Applications," Proc. IEEE, vol. 105, no. 6, pp. 990-1007, Jun. 2017, doi: 10.1109/JPROC.2017.2650235.

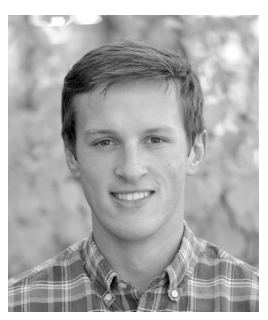

John Garrett received a BSc degree in electrical engineering from the University of Alberta in 2012, an MSc degree in electrical engineering from the University of Calgary in 2014, and a DPhil degree in astrophysics from the University of Oxford in 2018. He is currently a Submillimeter Array (SMA) Postdoctoral Fellow at the Center for Astrophysics Harvard \& Smithsonian. His research is focused on developing wide bandwidth SIS mixers, focal plane arrays and software for simulating SIS junctions.

Hawal Rashid Biography text here.

Ghassan Yassin Biography text here.

Vincent Desmaris Biography text here.

Alexey Pavolotsky Biography text here.

Victor Belitsky Biography text here. 